\title{
Competencias Comunicativas Interculturales en la formación inicial docente: El caso de tres universidades regionales de Chile*
}

\author{
Intercultural Communication Competencies in initial teacher training: \\ The case of three regional universities in Chile
}

\author{
Susan Sanhueza Henríquez, ${ }^{a}$ Pascal Patrick Matzler ${ }^{b}$, Chuan-Chih Hsuc, \\ Julio Domínguez Maldonado ${ }^{\text {, Miguel Friz Carrillo }}$, Segundo Quintriqueo Millán ${ }^{f}$ \\ a Universidad Católica del Maule. Correo electrónico: assanhueza@ucm.cl \\ ${ }^{\mathrm{b} c \mathrm{~d}}$ Universidad Católica del Maule

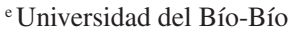 \\ ${ }^{\mathrm{f}}$ Universidad Católica de Temuco
}

\begin{abstract}
RESUMEN
El artículo tiene por objetivo conocer las habilidades cognitivas, afectivas y comportamentales que poseen los estudiantes de las carreras de Pedagogía en Educación Parvularia y Educación Básica $(n=270)$, para atender la diversidad sociocultural de futuros alumnos y a partir de ello, proponer elementos de la didáctica y pedagogía intercultural que deben aprender para trabajar en contextos escolares culturalmente diversos. Se optó por un enfoque cuantitativo de diseño descriptivo comparativo empleando la Escala de Inteligencia Cultural (The Cultural Intelligence Scale) con 22 ítems. Los resultados hacen aconsejable mejorar los currículos formativos de los futuros profesores integrando la temática de la diversidad cultural de manera explícita y transversal en las diferentes titulaciones, por ejemplo, a través del diseño e implementación de programas de desarrollo de la comunicación intercultural.
\end{abstract}

Palabras clave: diversidad cultural, formación inicial de profesores, comunicación intercultural

\begin{abstract}
The article aims to describe the cognitive, affective and behavioural competencies possessed by undergraduate students in initial teacher training courses for preschool and primary school $(n=270)$ to address the sociocultural diversity of their future students, and on this basis, to propose elements of intercultural didactics and pedagogy that these undergraduate students must learn in order to work in culturally diverse school settings. A quantitative approach of descriptive comparative design which makes use of the Cultural Intelligence Scale (CI) with 22 items was employed. The results make it advisable to improve the training curricula of future teachers by integrating the theme of cultural diversity explicitly and transversally in the different teacher training courses, for example, through the design and implementation of programs to develop intercultural communication.
\end{abstract}

Keywords: cultural diversity, initial teacher training, intercultural communication

Investigación financiada por la Comisión Nacional de Investigación Científica y Tecnológica de Chile, a través del Proyecto FONDECYT Regular N 1140864 titulado Desarrollo de la Competencia Comunicativa Intercultural (CCI) en estudiantes universitarios de las regiones del Maule, Bío-Bío y La Araucanía y por el proyecto Inmigrantes en el sistema educacional de Chile. Representaciones de género, lengua, territorialidad y exclusión social, del Programa de Investigación Asociativa ANILLOS DE INVESTIGACIÓN EN CIENCIAS SOCIALES Y HUMANIDADES, número SOC 1401. 
Estudios Pedagógicos XLII, N 4: 183-200, 2016

COMPETENCIAS COMUNICATIVAS INTERCULTURALES EN LA FORMACIÓN INICIAL DOCENTE: EL CASO DE TRES UNIVERSIDADES REGIONALES DE CHILE

\section{INTRODUCCIÓN}

El presente estudio se adscribe a la línea de investigación de la Competencia Comunicativa Intercultural (en adelante CCI), entendida como las habilidades cognitivas, afectivas y comportamentales que emplean los individuos culturalmente diferentes para favorecer un grado de comunicación eficaz, estableciendo relaciones apropiadas y efectivas en determinados contextos sociales y culturales. Así, surge la necesidad de explorar el desarrollo de la CCI en estudiantes universitarios de las regiones del Maule, Bío-Bío y La Araucanía. El interés de entregar una formación intercultural por parte del profesorado radica en una demanda cada vez más relevante a nivel nacional e internacional, debido a una realidad multicultural creciente en los países del mundo en el siglo XXI (Ragoonaden, 2011; Steinbach, 2012). Esta demanda no solo se sustenta en la incesante presencia de un alumnado culturalmente diverso en las instituciones escolares, sino también por la necesidad de dar una respuesta pedagógica de calidad, contextualizada a la realidad sociocultural de los estudiantes, lo cual plantea el desafío de construir escuelas inclusivas e interculturales (Leiva, 2012).

En América Latina han sido implementadas políticas de educación intercultural que han buscado avanzar en el reconocimiento y valoración de la diversidad cultural, lingüística y étnica, al interior de los sistemas educativos (Dietz y Mateos, 2013). Esto ha posibilitado la habilitación de programas especiales para la formación de profesores de origen indígena y no indígena, con el propósito de contar con profesionales capaces de entregar una educación intercultural contextualizada y coherente con las necesidades de los pueblos indígenas (Valiente, 2001; Gasché, 2013). Sin embargo, esta formación se ha limitado a la preparación de profesores para contextos indígenas, conservando una formación monocultural en los programas universitarios, asociados a tres condiciones: 1) por ser lo intercultural un campo de investigación y de prácticas recientes, que ha sido tratado de manera marginal en los sistemas educativos, en el medio social y universitario; 2) por persistir una lógica social y política dominante que busca conservar la monoculturalidad en las prácticas educativas; y 3) por la falta de voluntad de la estructura sociopolítica para confrontar el racismo institucionalizado en los diferentes niveles del sistema educativo escolar (Dasen, 2002; Mampaey y Zanoni, 2015).

En el caso de Chile, los tres factores se han conjugado, provocando que el impacto y comprensión de la interculturalidad queden minorizados al interior del sistema educativo y universitario, lo que ha conducido a que la preparación de los profesores se caracterice por una comprensión insuficiente de las culturas presentes en la sala de clases (Akkari, 2006). Esta situación no es exclusiva de la realidad chilena, siendo observable en territorios donde habita población indígena y no indígena, y en los nuevos escenarios multiculturales que surgen por las poblaciones inmigrantes (Dion, 2007; Akkari, 2009, 2010).

En este contexto, el Ministerio de Educación de Chile (2003, 2005) ha declarado, a través de distintas herramientas técnicas y orientaciones curriculares, la necesidad de preparar a los sujetos para relacionarse en mundos distintos al propio, destacando los principios de democracia, igualdad de oportunidades, capacidades y la necesidad de currículos interculturales (Ouellet, 1991, 2002; Dietz y Mateo, 2011). Sin embargo, cuando los niños se incorporan a la educación escolar se enfrentan a un currículo monocultural que les genera problemas en el aprendizaje y en la comunicación intercultural en sujetos que pertenecen a culturas diversas (Sanhueza, Quintriqueo y Friz, 2014). 
En particular, investigaciones en el ámbito de la formación de profesores (Jordán 1996, López, 2006, Quilaqueo y Quintriqueo, 2010) han llegado a identificar una serie estereotipos, destacando como los más frecuentes la idea de que las culturas minoritarias de los alumnos suponen un déficit o hándicap. El supuesto es que aún reconociendo la diferencia cultural de sus alumnos en las aulas, el profesorado no incorpora cambios en las prácticas profesionales y predomina un saber y conocimiento hegemónico, donde no se acepta otra lógica de conocimiento que no sea la occidental.

Como se ha planteado, las interacciones que se producen entre interlocutores culturalmente diversos puede tener una serie de connotaciones desde el ámbito educativo, especialmente, desde la formación inicial de profesores, ya que existen evidencias que indican que los estudiantes ponen en juego una serie de concepciones sobre el valor de la diversidad basada en su propia experiencia y en la interpretación de su propio proceso formativo (Lipiansky, 1992).

Entonces, cuando se produce un encuentro intercultural, los intervinientes interactúan apoyándose en suposiciones culturales propias (dimensión personal de la comunicación) que funcionan, según Schütz (1979), como pantallas perceptuales de los mensajes que intercambian, es decir, como modos de ver y comprender el mundo. De ahí que las experiencias intencionadas en los currículos de formación de futuros profesores debieran integrar elementos conceptuales y didácticos de la enseñanza y aprendizaje de la competencia comunicativa intercultural. Cabrera et al. (1999) propusieron un modelo de aprendizaje de las competencias interculturales bajo dos principios: a) formación en educación multicultural antes de la intervención; y b) integración de los contenidos con metodologías y estrategias de enseñanza.

Los ámbitos que integran este modelo de formación serían los siguientes:

De la competencia cognitiva destacan habilidades como conocer minorías étnicas del contexto real, conocer modelos y enfoques de educación intercultural, reflexionar sobre las condiciones necesarias para el diseño de un proyecto educativo global. El compromiso con una filosofía multicultural integra habilidades como el reconocimiento del otro, el análisis de las políticas educativas, reflexión sobre el currículo oculto del profesorado y como éste afecta a las minorías étnicas y la aceptación de los valores y actitudes de una educación intercultural. El desarrollo de actitudes y valores lleva a que el profesorado perciba la diversidad desde la diferencia, no desde el déficit; implica conocer las culturas, las subjetividades e intersubjetividades de los sujetos culturalmente diversos y considerar contextos multiculturales de sus alumnos.

En relación con las competencias pedagógicas, se deberían elaborar diseños curriculares y unidades didácticas de inspiración intercultural, usar diferentes metodologías y técnicas de enseñanza y aprendizaje (aprendizaje cooperativo), desarrollar estrategias de comunicación con las familias y otros profesionales, adoptar una actitud crítica para evaluar materiales de enseñanza y habilidades para diagnosticar los valores, necesidades educativas y estilos de aprendizaje del alumnado (Lázár et al., 2007; Lacourse y Maubant, 2009). Ouellet (2002) identifica cuatro componentes para la formación intercultural del profesorado en contextos interculturales: 1) la definición de un enfoque educativo; 2) explorar, conocer y reconocer culturas extranjeras a la propia en el contexto escolar; 3) la intervención del docente es un factor determinante en la calidad de los procesos educativos; y 4) el desarrollo de competencias específicas para trabajar en contextos de diversidad sociocultural. 
En relación al primer componente, la formación del profesorado necesita definir un enfoque educativo como marco referencial para lograr una comprensión mínima del pluralismo cultural del cual provienen sus estudiantes. Esto implica de parte del profesorado un conocimiento sobre conceptos clave como la cultura, etnicidad e identidad en contexto de modernidad y relativismo cultural. Asimismo, tomar conciencia de los obstáculos de las relaciones interculturales, como los prejuicios, la discriminación, el racismo y los problemas de la monoculturalidad (Quintriqueo, Quilaqueo, Gutiérrez, Morales y Sanhueza, 2015). Este marco conceptual y referencial para la acción pedagógica requiere ser lo más amplio y flexible posible, para establecer una perspectiva crítica respecto de sus acciones, con el objeto de redefinir sus concepciones y percepciones, desde un pluralismo epistemológico, para lograr nuevas comprensiones de los procesos pedagógicos contextualizados a la realidad sociocultural de sus estudiantes (Quilaqueo, Quintriqueo, Torres y Muñoz, 2014).

En relación al segundo componente, destacamos la necesidad de que el futuro profesorado tenga la capacidad de explorar, conocer y reconocer culturas extranjeras a la propia en el aula, no como una condición de la formación intercultural, sino más bien, como una condición de modificación y transformación de la sociedad postmoderna (Quintriqueo, Quilaqueo, Gutiérrez, Morales y Sanhueza, 2015). Este viaje por la diversidad cultural podría contribuir de manera significativa al desarrollo de una visión planetaria con respecto a la necesidad de confrontar marcos de referencias sociales y culturales, para lograr el aprendizaje y contextualizar los procesos educativos.

En relación al tercer componente, la intervención del docente es un factor determinante en la calidad de los procesos educativos con estudiantes que pertenecen a una diversidad sociocultural, puesto que es él quien les ofrece andamiajes eficientes, reconociendo, en cada uno de ellos, sus propias características y experiencias previas, logrando así desarrollar nuevas capacidades, habilidades y conocimientos (Quintriqueo, Quilaqueo, Gutiérrez, Morales y Sanhueza, 2015). En este sentido, es de vital importancia una intervención educativa eficaz, fortaleciendo el pluralismo epistemológico en la definición de la acción y finalidad educativa en contextos interculturales (Quilaqueo et al., 2014). En este proceso se identifican como ideas centrales la apertura a la colaboración escuela-familia-comunidad y la capacidad de autocrítica para redefinir las finalidades educativas, con el fin responder a la diversidad cultural, la igualdad, la equidad y la cohesión social (Quintriqueo et al., 2015).

En relación al cuarto componente, el desarrollo de competencias específicas para trabajar en contextos de diversidad sociocultural es de vital importancia para la formación de profesores que quisieran desarrollar una educación cada vez más intercultural (Ouellet, 2002). Algunas competencias específicas deseables de desarrollar en la formación inicial de profesores son:

a) Competencias para juzgar de manera crítica los componentes de la formación intercultural.

b) Competencias para estudiar y sistematizar la cultura de sus estudiantes, características de las familias y aspectos del medio sociocultural, para interpretar y aplicar estrategias de colaboración escuela-familia-comunidad.

c) Competencias necesarias para descubrir problemas de aprendizaje en grupo, en cooperación, con el objeto de proponerse clases que favorezcan un aprendizaje de 
alto nivel, desarrollando estrategias múltiples, ajustado al contexto sociocultural de sus estudiantes.

d) Competencias necesarias para abordar los conflictos y desacuerdos en relación a los valores educativos, desde una deliberación democrática, mediación intercultural, negociación y acomodación emocional, intelectual y comportamental razonable de los estudiantes, respetando las diferencias culturales.

e) Competencias necesarias para elaborar en conjunto con los colegas, criterios para evaluar el carácter democrático de la gestión, los contenidos interculturales de los programas, procesos de evaluación y orientación que consideren la diversidad cultural como los modos de valorar una conducta, un saber, la expresión oral, la presencia de estereotipos y actuación racista en forma explícita o implícita en los materiales educativos, el clima intercultural de la escuela y en el aula (Quintriqueo, Quilaqueo, Gutiérrez, Morales y Sanhueza, 2015).

Como se puede observar, la presencia o no de determinadas competencias interculturales en los futuros profesores sería determinante para la inclusión de escolares, especialmente, para aquellos que provienen de contextos sociales y culturales diferentes. En este contexto, nos preguntamos qué habilidades cognitivas, afectivas, comportamentales y pedagógicas poseen los estudiantes de las carreras de Pedagogía en Educación Parvularia y Educación Básica, para atender la diversidad sociocultural de sus futuros alumnos y cuáles son los elementos de la didáctica y pedagogía intercultural que deben aprender para trabajar en contextos escolares culturalmente diversos.

\section{MÉTODO}

\subsection{ENFOQUE Y DISEÑO}

Para dar respuesta a las preguntas de investigación se empleará un diseño no experimental descriptivo mediante encuesta. Lozada y López (2003) indican que los estudios no experimentales son más apropiados cuando la investigación se desarrolla en escenarios educativos naturales y lo que se pretende es conocer o descubrir una realidad. La investigación por encuesta consiste en un proceso de recolección de datos y de su descripción con el objetivo de dar respuesta a preguntas de interés. Según Cardona (2002), los procedimientos de investigación mediante encuesta establecen reglas que nos permiten acceder de forma científica a lo que las personas conocen, opinan o sienten, aspecto que resulta coherente con las dimensiones de la CCI a evaluar (dimensión cognitiva, afectiva y comportamental). Teóricamente, se asume que una competencia no es una cuestión de todo o nada, sino que existen grados entre estos polos que configuran un continuo actitudinal. Aquí surge la importancia de emplear como alternativa una escala de valoración Likert que permitirá inferir, a partir de ciertas respuestas verbales o no verbales del sujeto, las actitudes hacia las diferencias culturales que no son directamente observables. 


\subsection{CONTEXTO Y PARTICIPANTES}

El estudio se llevó a cabo en tres universidades regionales (Universidad Católica del Maule, Universidad del Bío-Bío y Universidad Católica de Temuco), todas pertenecientes al Consejo de Rectores de Universidades Chilenas (CRUCH). En particular, se optó por un muestreo no probabilístico de tipo disponible (Cornejo, Sanhueza y Rioseco, 2011), con la participación de 270 estudiantes de pedagogía de las regiones del Maule (42\%), Bío-Bío (29\%) y La Araucanía (29\%) de las carreras de Educación Parvularia (41\%) y Educación General Básica (59\%). Las edades fluctuaban entre 20 y 41 años $(M=23)$, con una presencia mayoritaria de mujeres $(90 \%)$. Es importante destacar que un porcentaje significativo de estudiantes señala provenir de zonas rurales (30\%) y un grupo menor pertenece a pueblos originarios $(8 \%)$. Respecto de la experiencia con culturas diferentes, los estudiantes declaran tener amigos que provienen de zonas rurales (94\%), del extranjero (46\%) y de pueblos originarios (61\%), por lo cual, su experiencia intercultural es significativa.

\subsection{VARIABLES E INSTRUMENTO}

La Competencia Comunicativa Intercultural (CCI) se midió mediante The Cultural Intelligence Scale (Ang, Van Dyne, Koh, Yee, Templer, Tay \& Chandrasekar, 2007), traducido y adaptado por los autores para ser empleado con estudiantes universitarios de Chile. La parte I contenía variables demográficas como la región de origen, universidad, edad, género y experiencia intercultural.

La parte II incluía la Escala de Inteligencia Cultural, a cuyos 22 reactivos se respondía expresando el grado de acuerdo utilizando una escala tipo Lickert $(1=$ Muy en desacuerdo, 2 $=$ En desacuerdo, $3=$ Ni de acuerdo ni en desacuerdo, $4=$ De acuerdo, $5=$ Muy de acuerdo). Luego de un análisis psicométrico del instrumento se obtuvo en la prueba de esfericidad de Bartlett $c^{2}(351)=3447,26 ; p<.000$ considerado como muy bueno para el estudio al igual que la prueba de adecuación de la muestra de Kaiser-Meyer-Olkin (KMO=0,88).

Los análisis de fiabilidad como consistencia interna arrojan un Alpha de Cronbach de 0.88 , lo que implica que las puntuaciones tienen un error muy pequeño y son altamente fiables.

Posteriormente, se empleó el método de componentes principales como método de extracción de factores con autovalores mayores a la unidad obteniendo una solución factorial de cuatro factores que explican el $53 \%$ de la varianza: Dimensión Pedagógica (17\%) con siete ítems y la Dimensión Comportamental (14\%), Dimensión Afectiva (13\%) y Dimensión Cognitiva (9\%) con cinco ítems en cada una.

En la rotación varimax, se observa que todas las variables quedan bien representadas con valores de la comunalidad que van desde 0.5 a 0.8 con una carga relativamente homogénea.

Es importante señalar que el instrumento original estaba construido sobre la base de cuatro dimensiones: Congnitiva, Afectiva, Comportamental y Metacognitiva. No obstante, los análisis psicométricos no confirmaron esta última dimensión con la población estudiada. Adicionalmente, en nuestro trabajo hemos incluido la Dimensión Pedagógica por tratarse de una evaluación de futuros profesores. Esta dimensión fue confirmada con los análisis y pruebas estadísticas.

Los cuatro factores encontrados responden a diferentes dimensiones que quedan definidas por las variables que las integran: 


\section{Factor I Dimensión Cognitiva}

Enfatiza el cambio del pensamiento individual sobre el entorno, a través de la comprensión de las características distintivas de uno(a) mismo(a) y de otras culturas. Se trata del proceso de reducción del nivel de ambigüedad situacional y de incertidumbre en las interacciones interculturales (Chen y Starosta, 1996). Se promueve la oportunidad de desarrollar la conciencia de las dinámicas culturales mediante un doble aspecto de comprensión de la realidad: la autoconciencia y la conciencia cultural.

\section{Factor II Dimensión Comportamental}

Este factor integra un conjunto de destrezas específicas para la solución de problemas o la formación de relaciones, que les permitiera interactuar e intercambiar significados entre miembros de culturas diferentes. En concreto, se entiende como el conjunto de habilidades verbales y no verbales que evidencian una adaptación de la conducta que favorece la comunicación eficaz, destacando la flexibilidad del comportamiento y el manejo de la interacción, entre otras.

\section{Factor III Dimensión Afectiva}

Se centra en las emociones personales o los cambios de sentimientos causados por situaciones particulares, personas y ambientes. Una persona competente interculturalmente es capaz de proyectar y recibir respuestas emocionales positivas antes, durante y después de las interacciones interculturales.

\section{Factor IV Dimensión Pedagógica}

Integra todas aquellas habilidades necesarias para educar la CCI. En particular, se enmarca en el rol mediador del profesor (o futuro profesor) e incluye competencias referidas a la planificación, evaluación y didáctica intercultural.

\subsection{PROCEDIMIENTO Y ANÁLISIS DE DATOS}

El cuestionario fue aplicado por tres encuestadores que informaron a los participantes sobre los objetivos del estudio. Los estudiantes firmaron previamente un consentimiento informado. Se destacó que la participación tenía un carácter voluntario, confidencial y anónimo. Durante la aplicación los encuestadores apoyaron a los estudiantes que lo necesitaron. El tiempo estimado de respuesta fue de 30 minutos.

Respecto del análisis de datos, se utilizó la estadística descriptiva (descriptivos, frecuencias, porcentajes y medidas de dispersión). Para realizar los análisis diferenciales (por género, universidad, titulación y experiencia intercultural) se realizó la prueba $t$ de Student para muestras independientes. Los análisis fueron apoyados del software estadístico SPSS 18.0.

\section{RESULTADOS}

En un primer análisis global de las respuestas, es posible concluir que la dimensión más desarrollada en los estudiantes es la afectiva, vale decir, son capaces de hacer uso de sus experiencias previas emitiendo respuestas emocionales positivas frente a personas culturalmente diferentes (Ver Figura 1). 
Figura 1. Medias obtenidas en las respuestas de los estudiantes universitarios por dimensión

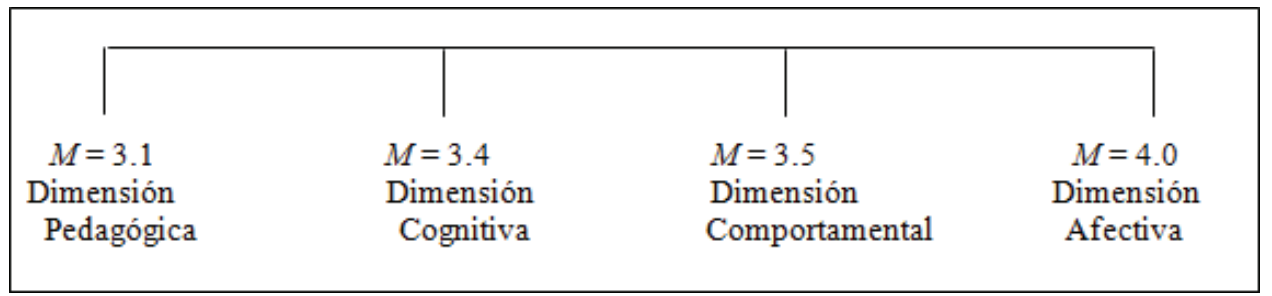

De interés para la formación inicial de profesores es que la dimensión menos desarrollada no sea precisamente la que mide las competencias interculturales sino la dimensión pedagógica que supone las formas de enseñar a través de un currículo culturalmente diverso. En efecto, los estudiantes consideran que las competencias para planificar, evaluar y/o adaptar estrategias metodológicas a la diversidad cultural de los escolares no ha sido educada por las universidades.

De igual manera, si diferenciamos las respuestas en función de las universidades, los resultados confirman estudios previos que señalan que a mayor diversidad cultural, mayor desarrollo de competencias interculturales (Ver Tabla 1).

Quizás resulte conveniente puntualizar que para el estudio hemos empleado el concepto de diversidad cultural de Barra (2007) que hace referencia a los diferentes significados o interpretaciones que distintos grupos existentes en Chile tienen acerca de su propia existencia y experiencias en la sociedad. Incluye por lo tanto a pueblos originarios, población mestiza indígena -español, nuevos migrantes y personas provenientes de zonas rurales habitualmente en condición de aislamiento geográfico. Bajo este enfoque, las regiones del Bío-Bío y Maule concentran una alta población rural, mientras que La Araucanía una alta población mapuche.

Tabla 1. Medias obtenidas por dimensión y universidad

\begin{tabular}{|c|c|c|c|c|}
\hline Universidad & $\begin{array}{c}\text { Dimensión } \\
\text { Cognitiva }\end{array}$ & $\begin{array}{c}\text { Dimensión } \\
\text { Afectiva }\end{array}$ & $\begin{array}{c}\text { Dimensión } \\
\text { Comportamental }\end{array}$ & $\begin{array}{c}\text { Dimensión } \\
\text { Pedagógica }\end{array}$ \\
\hline $\begin{array}{c}\text { Universidad región } \\
\text { del Bío-Bío }\end{array}$ & 3.2 & 3.9 & 3.4 & 2.5 \\
\hline $\begin{array}{c}\text { Universidad región } \\
\text { del Maule }\end{array}$ & 3.4 & 4.0 & 3.7 & 3.2 \\
\hline $\begin{array}{c}\text { Universidad región } \\
\text { La Araucanía }\end{array}$ & 3.6 & 4.2 & 3.2 & 3.5 \\
\hline
\end{tabular}

Como se puede observar, las puntuaciones más altas son obtenidas por la universidad localizada geográficamente en la región de La Araucanía. Esto podría estar asociado a que en dicha región, la universidad participante incorpora en algunas carreras la interculturalidad y la educación intercultural como un eje central de la formación inicial docente. También es 
posible poner en discusión otras posibles explicaciones para estas diferencias, por ejemplo, que los estudiantes suelen no asociar la diversidad cultural con personas que habitan en zonas rurales, o que los currículos formativos incluyen en mayor o menor medida cursos o contenidos referidos a la educación intercultural. De cualquier manera, pensamos que la concepción de diversidad cultural que poseen los estudiantes de pedagogía determina la forma de aproximarse a prácticas más o menos inclusivas. Asimismo, determina las respuesta educativas que se supone implementarán en el aula, lo que les llevará a diversificar o no las estrategias de enseñanza y aprendizaje en función de los contextos socioculturales.

En un análisis por dimensión, los resultados muestran que las competencias más desarrolladas en la dimensión cognitiva (Ver Tabla 2) son el conocimiento de las artes $(61 \%)$, los valores y creencias de otras culturas (56\%) y los rituales y ceremonias (49\%).

Tabla 2. Descriptivos de dimensión cognitiva de la CCI

\begin{tabular}{|lccccccc|}
\hline \multicolumn{1}{|c}{ Dimensión cognitiva } & \multicolumn{7}{cc}{$\%$} \\
\cline { 2 - 8 } & $M$ & $D T$ & $M D$ & $D$ & $N A D$ & $D A$ & $M A$ \\
\hline Conozco cómo funcionan a nivel político otras culturas & & & & & & & \\
Toda la muestra & 2.9 & 1.0 & 10 & 19 & $\underline{38}$ & 31 & 2 \\
$\quad$ Universidad región del Bío-Bío & 2.8 & 1.0 & 13 & 17 & $\underline{41}$ & 28 & 1 \\
Universidad región del Maule & 3.0 & 0.8 & 6 & 19 & $\underline{43}$ & 31 & 1 \\
Universidad región La Araucanía & 2.9 & 1.1 & 14 & 20 & $\underline{27}$ & 34 & 4 \\
\hline Conozco las reglas de otras lenguas o idiomas & & & & & & & \\
Toda la muestra & 3.0 & 1.1 & 13 & 14 & 35 & 33 & 5 \\
Universidad región del Bío-Bío & 2.7 & 1.1 & 22 & 11 & 40 & 22 & 5 \\
Universidad región del Maule & 3.2 & 1.0 & 9 & 15 & 32 & 38 & 5 \\
Universidad región La Araucanía & 3.1 & 1.0 & 9 & 17 & 33 & 37 & 4 \\
\hline Conozco los valores y creencias de otras culturas & & & & & & & \\
Toda la muestra & 3.7 & 0.9 & 2 & 7 & 23 & $\underline{56}$ & 12 \\
Universidad región del Bío-Bío & 3.5 & 0.9 & 4 & 10 & 25 & 50 & 10 \\
Universidad región del Maule & 3.6 & 0.8 & 3 & 9 & 20 & $\underline{61}$ & 7 \\
Universidad región La Araucanía & 3.9 & 0.7 & 0 & 3 & 23 & 54 & 20 \\
\hline Conozco los ritos o ceremonias que realizan otras culturas & & & & & & \\
Toda la muestra & 3.5 & 1.0 & 5 & 10 & 23 & $\underline{49}$ & 13 \\
Universidad región del Bío-Bío & 3.3 & 1.0 & 8 & 10 & 29 & 45 & 8 \\
Universidad región del Maule & 3.4 & 1.0 & 7 & 12 & 24 & 46 & 10 \\
Universidad región La Araucanía & 3.9 & 0.7 & 0 & 6 & 15 & $\underline{58}$ & 20 \\
\hline Conozco las artes de otras culturas & & & & & & & \\
Toda la muestra & 4.0 & 0.8 & 1 & 4 & 10 & $\underline{61}$ & 24 \\
Universidad región del Bío-Bío & 4.0 & 0.6 & 0 & 3 & 6 & $\underline{72}$ & 19 \\
Universidad región del Maule & 3.9 & 0.7 & 0 & 7 & 13 & 60 & 20 \\
Universidad región La Araucanía & 4.1 & 0.9 & 4 & 1 & 8 & 51 & 36 \\
\hline
\end{tabular}

$M D=$ Muy en Desacuerdo, $D=$ En desacuerdo, $N A D=$ Ni de acuerdo ni en desacuerdo, $D A=$ De acuerdo, $M A=M u y$ de acuerdo 
Estudios Pedagógicos XLII, N 4: 183-200, 2016

COMPETENCIAS COMUNICATIVAS INTERCULTURALES EN LA FORMACIÓN INICIAL DOCENTE: EL CASO DE TRES UNIVERSIDADES REGIONALES DE CHILE

En particular, los estudiantes de la universidad de la región del Maule han incorporado conocimientos sobre valores y creencias de otras culturas, la universidad de la región de La Araucanía destaca por el aprendizaje de ritos y ceremonias que realizan culturas diferentes en el marco de sus planes de formación inicial. Mientras que la universidad de la región del Bío-Bío presenta un mayor desarrollo de competencias relacionadas con el conocimiento del arte en su proceso de formación inicial. En tanto, la universidad que obtiene mejores resultados en esta dimensión cognitiva es la ubicada en la región de La Araucanía, seguido por Maule y Bío-Bío, respectivamente.

Un elemento importante de hacer notar en esta dimensión son las respuestas en el ítem de conocimiento de cómo funcionan a nivel político, normativo o social las diferentes culturas, donde los estudiantes de las tres universidades concentraron sus respuestas en la opción ni de acuerdo ni en desacuerdo (neutralidad), lo que podría estar referido a una falta de conocimiento en la materia. Estas respuestas, al menos dubitativas, son similares para la pregunta que consulta aspectos referidos al conocimiento de otras lenguas.

En relación con la dimensión afectiva (Ver Tabla 3), nuevamente la universidad de La Araucanía obtiene la Media más alta $(M=4.1)$, luego la universidad del Maule $(M=$ 3.9) y Bío-Bío $(M=3.9)$, sin embargo, en esta dimensión todas las universidades obtienen sus puntuaciones más altas develando un desarrollo más homogéneo en cada habilidad evaluada. Destacan el disfrute de la interacción $(M=4.4)$ y la motivación por conocer nuevas culturas $(M=4.6)$, solo se obtienen porcentajes más bajos en los ítem asociados a la confianza $(M=3.8)$ y seguridad en las propias capacidades para relacionarse con personas de otras culturas $(M=3.6)$.

Tabla 3. Descriptivos de dimensión afectiva de la CCI

\begin{tabular}{|llllllll|}
\hline \multicolumn{1}{|c}{ Dimensión afectiva } & \multicolumn{7}{cc}{$\%$} \\
\cline { 2 - 8 } & M & DT & MD & D & NAD DA & MA \\
\hline Disfruto de la interacción con personas de diferentes cul- & & & & & & \\
turas & 4.4 & 0.8 & 2 & 1 & 10 & 36 & $\underline{52}$ \\
$\quad$ Toda la muestra & 4.2 & 0.8 & 1 & 0 & 15 & 37 & $\underline{46}$ \\
$\quad$ Universidad región del Bío-Bío & 4.3 & 0.8 & 1 & 1 & 10 & 39 & $\underline{48}$ \\
$\quad$ Universidad región del Maule & 4.4 & 0.9 & 4 & 1 & 2 & 28 & $\underline{64}$ \\
$\quad$ Universidad región La Araucanía & & & & & & \\
\hline Confío en mis habilidades para interactuar con personas & & & & & & \\
culturalmente diferentes & 3.8 & 0.9 & 2 & 9 & 16 & 53 & 22 \\
$\quad$ Toda la muestra & 3.7 & 1.0 & 4 & 9 & 22 & 43 & 22 \\
$\quad$ Universidad región del Bío-Bío & 3.8 & 0.9 & 2 & 9 & 14 & 58 & 17 \\
$\quad$ Universidad región del Maule & 4.0 & 0.8 & 0 & 8 & 11 & 54 & 27 \\
$\quad$ Universidad región La Araucanía & & & & & & \\
\hline Me siento seguro de mi capacidad para relacionarme con & & & & & & \\
personas de otras culturas & 3.6 & 1.1 & 4 & 16 & $\underline{19}$ & 44 & 18 \\
$\quad$ Toda la muestra & 3.4 & 1.1 & 6 & 19 & $\underline{19}$ & 37 & 18 \\
$\quad$ Universidad región del Bío-Bío & 3.5 & 1.0 & 4 & 17 & $\underline{17}$ & 46 & 15 \\
$\quad$ Universidad región del Maule & 3.7 & 0.9 & 0 & 10 & $\underline{22}$ & 46 & 22 \\
\hline Universidad región La Araucanía & & & & &
\end{tabular}




\begin{tabular}{|lccccccc|}
\hline Disfruto el hecho de conocer nuevas culturas & 4.6 & 0.7 & 2 & 0 & 4 & 26 & $\underline{69}$ \\
$\quad$ Toda la muestra & 4.6 & 0.7 & 1 & 0 & 6 & 21 & $\underline{\underline{72}}$ \\
$\quad$ Universidad región del Bío-Bío & 4.5 & 0.7 & 1 & 1 & 5 & 31 & $\underline{\mathbf{6 2}}$ \\
$\quad$ Universidad región del Maule & 4.6 & 0.7 & 3 & 0 & 0 & 23 & $\underline{\mathbf{7 4}}$ \\
$\quad$ Universidad región La Araucanía & & & & & & & \\
\hline Confío que puedo comprar (comida, ropa, etc.) a perso- & & & & & & & \\
nas de otras culturas & 4.0 & 1.0 & 2 & 9 & $\underline{16}$ & 40 & 34 \\
$\quad$ Toda la muestra & 3.9 & 0.9 & 0 & 9 & $\underline{18}$ & 46 & 27 \\
$\quad$ Universidad región del Bío-Bío & 3.8 & 1.0 & 3 & 10 & $\underline{14}$ & 47 & 25 \\
$\quad$ Universidad región del Maule & 4.2 & 0.9 & 1 & 5 & $\underline{15}$ & 25 & 53 \\
$\quad$ Universidad región La Araucanía & & & & &
\end{tabular}

$M D=$ Muy en Desacuerdo, $D=$ En desacuerdo, $N A D=$ Ni de acuerdo ni en desacuerdo, $D A=$ De acuerdo, $M A=$ Muy de acuerdo

Respecto de la dimensión comportamental (Ver Tabla 4), se puede observar que los estudiantes de pedagogía emplean diversas estrategias para relacionarse con personas culturalmente diferentes. Estas van desde el lenguaje verbal $(M=3.6)$ y no verbal $(M=$ 3.7), hasta la modificación del ritmo de la conversación $(M=3.7)$ o la modificación de expresiones faciales $(M=3.0)$.

Tabla 4. Descriptivos de dimensión comportamental de la CCI

\begin{tabular}{|c|c|c|c|c|c|c|c|}
\hline \multirow{2}{*}{ Dimensión comportamental } & \multicolumn{7}{|c|}{$\%$} \\
\hline & M & DT & MD & D & NAD & DA & MA \\
\hline \multicolumn{8}{|c|}{$\begin{array}{l}\text { Cambio mi comportamiento verbal (por ejemplo tono de } \\
\text { voz, acento) cuando una comunicación intercultural lo } \\
\text { requiere }\end{array}$} \\
\hline Toda la muestra & $\underline{3.6}$ & 1.0 & 5 & 10 & 22 & 48 & 15 \\
\hline Universidad región del Bío-Bío & 3.5 & 0.9 & 4 & 8 & 27 & 50 & 11 \\
\hline Universidad región del Maule & 3.8 & 0.9 & 2 & 7 & 20 & 49 & 21 \\
\hline Universidad región La Araucanía & 3.2 & 1.1 & 10 & 15 & 19 & 45 & 10 \\
\hline \multicolumn{8}{|c|}{$\begin{array}{l}\text { Utilizo la pausa y el silencio de manera diferente para } \\
\text { adaptarme a situaciones interculturales }\end{array}$} \\
\hline Toda la muestra & 3.5 & 1.0 & 6 & 11 & 25 & 44 & 14 \\
\hline Universidad región del Bío-Bío & 3.5 & 0.9 & 4 & 11 & 28 & 45 & 11 \\
\hline Universidad región del Maule & 3.7 & 0.8 & 1 & 9 & 25 & 50 & 16 \\
\hline Universidad región La Araucanía & 3.2 & 1.2 & 14 & 13 & 23 & 34 & 15 \\
\hline \multicolumn{8}{|c|}{$\begin{array}{l}\text { Modifico el ritmo de mi conversación (hablo más rápido } \\
\text { o más lento) cuando una situación intercultural lo re- } \\
\text { quiere }\end{array}$} \\
\hline Toda la muestra & $\underline{3.7}$ & 1.0 & 4 & 8 & 20 & $\underline{47}$ & $\underline{20}$ \\
\hline Universidad región del Bío-Bío & 3.7 & 0.8 & 1 & 6 & 24 & $\underline{51}$ & 17 \\
\hline Universidad región del Maule & 3.8 & 0.9 & 2 & 8 & 19 & $\underline{45}$ & $\underline{25}$ \\
\hline Universidad región La Araucanía & 3.4 & 1.2 & 11 & 9 & 18 & $\underline{46}$ & $\underline{15}$ \\
\hline
\end{tabular}


Estudios Pedagógicos XLII, $\mathrm{N}^{\circ}$ 4: 183-200, 2016

COMPETENCIAS COMUNICATIVAS INTERCULTURALES EN LA FORMACIÓN INICIAL DOCENTE: EL CASO DE TRES UNIVERSIDADES REGIONALES DE CHILE

\begin{tabular}{|lllllllll|}
\hline $\begin{array}{l}\text { Cambio mi comportamiento no verbal (gestos, uso de } \\
\text { manos y cuerpo) cuando una situación intercultural lo } \\
\text { requiere }\end{array}$ & & & & & & & \\
$\quad$ Toda la muestra & $\underline{3.7}$ & 1.0 & 6 & 7 & 20 & 45 & 22 \\
$\quad$ & 3.6 & 0.9 & 5 & 5 & 26 & 47 & 17 \\
$\quad$ Universidad región del Bío-Bío & 3.9 & 0.9 & 3 & 5 & 16 & 47 & 28 \\
$\quad$ Universidad región del Maule & 3.3 & 1.2 & 11 & 11 & 22 & 38 & 16 \\
$\quad$ Universidad región La Araucanía & & & & & & & \\
\hline $\begin{array}{l}\text { Modifico mis expresiones faciales cuando enfrento una } \\
\text { situación comunicativa con alguien de otra cultura }\end{array}$ & $\underline{3.0}$ & 1.3 & 19 & 17 & 18 & 35 & 12 \\
$\quad$ Toda la muestra & 2.6 & 1.2 & 24 & 27 & 19 & 25 & 4 \\
$\quad$ Universidad región del Bío-Bío & 3.3 & 1.2 & 11 & 12 & 18 & 42 & 16 \\
$\quad$ Universidad región del Maule & 3.0 & 1.4 & 23 & 13 & 15 & 34 & 14 \\
$\quad$ Universidad región La Araucanía & & & & & & & & \\
\hline
\end{tabular}

$M D=$ Muy en Desacuerdo, $D=$ En desacuerdo, $N A D=$ Ni de acuerdo ni en desacuerdo, $D A=$ De acuerdo, $M A=M u y$ de acuerdo

Si bien en las otras dimensiones la universidad localizada en La Araucanía obtenía mayores puntuaciones, este no es el caso, ya que es la universidad de la región del Maule la que obtiene mejores resultados. En este contexto, es posible inferir que si bien los participantes que estudian en contextos multiculturales parecieran estar dotados de herramientas técnicas, conceptuales y actitudes más inclusivas, a la hora de actuar reconocen no contar con habilidades que le permitan desenvolverse adecuadamente en contextos comunicativos interculturales.

En relación con la dimensión pedagógica (Ver Tabla 5), es necesario mencionar que fue integrada a partir de las características que tenía el grupo participante del estudio, es decir, estudiantes de pedagogía de los últimos años de carrera y que en consecuencia debía tener instaladas ciertas competencias en materia de diversidad cultural. A partir de los resultados es posible señalar que se trata de la competencia menos desarrollada en los estudiantes en las tres universidades.

Tabla 5. Descriptivos de dimensión pedagógica de la CCI

\begin{tabular}{|lcccccccc|}
\hline \multicolumn{1}{|c}{ Dimensión pedagógica } & \multicolumn{1}{c}{$\%$} & & & \\
\hline & $M$ & DT & MD & D & NAD DA & MA \\
\hline $\begin{array}{l}\text { En mi formación pedagógica he recibido conocimiento } \\
\text { teórico sobre la competencia comunicativa intercultural }\end{array}$ & & & & & & & \\
$\quad$ Toda la muestra & 2.8 & 1.3 & $\underline{18}$ & $\underline{26}$ & 20 & 26 & 10 \\
Universidad región del Bío-Bío & 2.5 & 1.2 & $\underline{24}$ & $\underline{32}$ & 17 & 22 & 5 \\
Universidad región del Maule & 2.9 & 1.2 & $\underline{14}$ & $\underline{27}$ & 22 & 28 & 9 \\
Universidad región La Araucanía & 3.1 & 1.3 & $\underline{17}$ & $\underline{19}$ & 20 & 27 & 16 \\
\hline
\end{tabular}




\begin{tabular}{|c|c|c|c|c|c|c|c|}
\hline $\begin{array}{l}\text { En mi formación pedagógica he } r \\
\text { metodológicas para trabajar la mu }\end{array}$ & & & & & & & \\
\hline Toda la muestra & 3.1 & 1.3 & 13 & 24 & 12 & 37 & 14 \\
\hline Universidad región del Bío-Bío & 2.5 & 1.2 & 25 & 31 & 14 & 23 & 6 \\
\hline Universidad región del Maule & 3.2 & 1.2 & 11 & 25 & 10 & 40 & 13 \\
\hline Universidad región La Araucanía & 3.7 & 1.0 & 4 & 14 & 13 & $\underline{46}$ & $\underline{23}$ \\
\hline $\begin{array}{l}\text { En mi formación pedagógica he rec } \\
\text { establecer relaciones afectivas en } \mathrm{s} \\
\text { dad cultural }\end{array}$ & & & & & & & \\
\hline Toda la muestra & 3.2 & 1.2 & 11 & 23 & 18 & 36 & 13 \\
\hline Universidad región del Bío-Bío & 2.6 & 1.2 & 19 & 32 & 18 & 25 & 5 \\
\hline Universidad región del Maule & 3.3 & 1.1 & 9 & 21 & 16 & $\underline{44}$ & $\underline{10}$ \\
\hline Universidad región La Araucanía & 3.5 & 1.1 & 5 & 15 & 22 & 34 & 23 \\
\hline $\begin{array}{l}\text { En mi formación pedagógica he } \mathbf{r} \\
\text { acerca del contexto sociocultural } \\
\text { diantes }\end{array}$ & & & & & & & \\
\hline Toda la muestra & 3.5 & 1.1 & 7 & 19 & 12 & 46 & 17 \\
\hline Universidad región del Bío-Bío & 2.9 & 1.3 & 18 & 23 & 13 & 35 & 11 \\
\hline Universidad región del Maule & 3.5 & 1.1 & 3 & 23 & 10 & 48 & 15 \\
\hline Universidad región La Araucanía & 3.9 & 0.8 & 0 & 8 & 14 & $\underline{52}$ & $\underline{25}$ \\
\hline $\begin{array}{l}\text { En mi formación pedagógica he } \mathbf{r} \\
\text { para planificar experiencias de } \\
\text { estudiantes de diversas culturas }\end{array}$ & & & & & & & \\
\hline Toda la muestra & 2.9 & 1.3 & $\underline{16}$ & $\underline{26}$ & 15 & 31 & 13 \\
\hline Universidad región del Bío-Bío & 2.4 & 1.2 & $\underline{24}$ & $\underline{36}$ & 17 & 17 & 6 \\
\hline Universidad región del Maule & 3.1 & 1.3 & $\underline{14}$ & $\underline{24}$ & 11 & 36 & 14 \\
\hline Universidad región La Araucanía & 3.3 & 1.2 & $\underline{9}$ & $\underline{18}$ & 19 & $\underline{37}$ & $\underline{17}$ \\
\hline $\begin{array}{l}\text { En mi formación pedagógica he rec } \\
\text { vinculación en contextos multic } \\
\text { pedagógicas) }\end{array}$ & & & & & & & \\
\hline Toda la muestra & 3.1 & 1.3 & 13 & $\underline{27}$ & 13 & 30 & 18 \\
\hline Universidad región del Bío-Bío & 2.5 & 1.2 & 23 & $\underline{35}$ & 13 & 20 & 9 \\
\hline Universidad región del Maule & 3.3 & 1.2 & 8 & $\underline{27}$ & 12 & 37 & 16 \\
\hline Universidad región La Araucanía & 3.5 & 1.3 & 9 & $\underline{20}$ & 13 & 28 & 29 \\
\hline $\begin{array}{l}\text { En mi formación pedagógica he } \mathbf{r} \\
\text { para adaptar las evaluaciones a } \\
\text { culturales de los estudiantes }\end{array}$ & & & & & & & \\
\hline Toda la muestra & 3.1 & 1.3 & 16 & 22 & 9 & 40 & 13 \\
\hline Universidad región del Bío-Bío & 2.4 & 1.3 & $\underline{34}$ & $\underline{26}$ & 9 & 26 & 5 \\
\hline Universidad región del Maule & 3.3 & 1.2 & 10 & 20 & 9 & $\underline{47}$ & $\underline{13}$ \\
\hline Universidad región La Araucanía & 3.5 & 1.1 & 4 & 20 & 10 & $\underline{45}$ & $\underline{20}$ \\
\hline
\end{tabular}

$M D=$ Muy en Desacuerdo, $D=$ En desacuerdo, $N A D=$ Ni de acuerdo ni en desacuerdo, $D A=$ De acuerdo, $M A=M u y$ de acuerdo 
Como se puede observar, un número significativo de estudiantes expresa no haber recibido formación en el ámbito de la competencia comunicativa intercultural (los porcentajes oscilan entre 14\% y 32\%). Los estudiantes de la universidad de La Araucanía expresan haber recibido conocimiento acerca del contexto sociocultural de sus futuros estudiantes (52\% y $25 \%$ de acuerdo, respectivamente). Por otra parte, la universidad de la región del Maule declara haber recibido estrategias didácticas para favorecer la diversidad cultural en el aula (44\% y 10\% de acuerdo respectivamente). En el ámbito de la evaluación, son los estudiantes de la región del Maule (47\% y 13\%) y La Araucanía (45\% y 20\%) quienes señalan haber recibido formación suficiente para adaptar las evaluaciones a diferentes contextos culturales. Los datos obtenidos permiten concluir que es la planificación para estudiantes de diversas culturas el nudo más crítico en la formación de futuros profesores de todas las instituciones participantes.

\section{DISCUSIÓN Y CONCLUSIONES}

Como señalábamos al inicio del trabajo, el propósito del estudio fue aproximarnos a las habilidades que presentaban futuros profesores para educar en contextos de diversidad cultural. A partir de los resultados, podemos concluir que la dimensión menos desarrollada por los estudiantes es la dimensión pedagógica, lo que nos indica las escasas herramientas que los estudiantes manifiestan tener para enseñar en contextos interculturales. Desde nuestra perspectiva, este es un resultado relevante por cuanto el estudio deja en tensión nuevamente la relación conocimiento pedagógico versus conocimiento disciplinar. En consecuencia, tendríamos que volver a preguntarnos cómo podrían los futuros profesores educar para la diversidad cultural si sus competencias en ámbitos relativos a la planificación o evaluación les resultan insuficientes. Esta pregunta engloba cuestiones mucho más complejas que la sola práctica educativa ya que encarna la comprensión de hombre y sociedad que el sistema educativo (en todos sus niveles) intenta validar.

No se trata solo de la superficialidad con que temas de diversidad cultural son expuestos en los programas de estudios, sino del predominio de una racionalidad monocultural que permea al sistema en su conjunto, de allí que la educación de la competencia comunicativa intercultural en la formación de los profesores continúe siendo un desafío. Si bien lo intercultural encarna principios éticos fundamentales, como el humanismo, la tolerancia o la hospitalidad, se constata que las transformaciones toman tiempo considerando la indiferencia que persiste sobre las diferencias en contextos políticos, sociales y culturales anclados a modelos republicanos, como es el caso de América Latina (Payet, 2006).

En el trabajo, los estudiantes manifestaron inseguridad frente a los conocimientos necesarios para enseñar en contextos multiculturales como, por ejemplo, el conocimiento de diferentes lenguas o formas de organización políticas de otras culturas. Este aspecto ya había sido documentado previamente por Gay y Howard (2000), quienes, observando el medio escolar estadounidense, indican que es común ver en los estudiantes de programas de formación de profesores una actitud temerosa (literalmente miedo) cuando corresponde enseñar la diversidad racial, étnica y cultural, especialmente con población indígena, inmigrantes y afrodescendientes. Es por ese motivo que proponemos, que la competencia comunicativa intercultural debe ser obligatoria, explícita y exhaustiva en la formación de profesores que habrán de desempeñarse en contextos interculturales. Esto implica formar 
a los futuros profesores en los conocimientos y habilidades, que les permitan transformar los programas y prácticas educativas que perpetúan el racismo y los prejuicios sobre la diversidad social y cultural, especialmente en contextos indígenas y de población inmigrantes en Chile.

Asimismo, los resultados evidencian que la experiencia intercultural pudiera ser un factor determinante para el aprendizaje de competencias comunicativas interculturales, aspecto confirmado en estudios previos (Ang et al., 2007; Aleksandrovna, 2014; Sanhueza et al., 2014). En el estudio, los futuros profesores que aprenden en contextos de diversidad cultural, reciben formación en educación intercultural y provienen de pueblos originarios obtienen mayores puntuaciones en las competencias evaluadas, especialmente, en la dimensión afectiva. No obstante, cuando se evalúa la dimensión comportamental estas puntuaciones tienden a disminuir. Entonces, habría que preguntarse si la formación está privilegiando competencias de tipo afectivas y cognitivas, en desmedro de las comportamentales y, si es necesario, proponer estrategias a nivel formativo que les permita a los estudiantes "poner en práctica” el conocimiento más bien teórico. El diseño de prácticas pedagógicas tempranas cuidadosamente diseñadas en contextos de diversidad cultural podría ser una herramienta efectiva para el aprendizaje de las competencias evaluadas. Muller et al. (2013) indican que las prácticas pedagógicas son una valiosa actividad formativa para integrar la formación disciplinar y didáctica. No obstante, existe un evidente distanciamiento entre los cursos universitarios y las prácticas de las escuelas. A partir de ello, estos autores proponen que la práctica incluya la participación de los estudiantes de pedagogía en las planificaciones, evaluaciones y tareas de los alumnos.

Como señalamos en la pregunta de investigación, también nos propusimos aportar con elementos de la didáctica y la pedagogía intercultural que deben aprender los estudiantes para trabajar en contextos escolares culturalmente diversos. Siguiendo los planteamientos de Jordán, Castellà y Pinto (2001), pensamos que la formación inicial de profesores demanda una clarificación conceptual acerca de las nociones principales de la teoría intercultural (como cultura, identidad cultural, integración, etnocentrismo, relativismo, etc.).

Para ello, proponemos como punto de partida ampliar el concepto de diversidad cultural (pueblos originarios, población mestiza indígena-español, nuevos migrantes y personas provenientes de zonas rurales habitualmente en condición de aislamiento geográfico, entre otros). Es importante que los estudiantes relativicen el concepto de cultura, esto con el propósito de realizar una adecuada valoración y reconocimiento de todas las culturas. Contrariamente, nuestros resultados muestran que los participantes del estudio solo comprendían la educación intercultural para el caso de pueblos originarios, negando así la educación intercultural para hijos de migrantes o estudiantes provenientes de zonas rurales o de aislamiento geográfico que poseen una enorme riqueza cultural y formas de comprender la realidad distintas. En este contexto, resulta necesario intencionar en el currículum experiencias de alumnos de diferentes orígenes culturales, con distintas lenguas y costumbres. Así, los futuros profesores se motivarán a plantearse un marco referencial para comprender el contexto sociocultural donde se desempeñan, nuevas actitudes y estrategias metodológicas para aprender la diversidad social y cultural de sus estudiantes (Quintriqueo, Quilaqueo, Gutiérrez, Morales y Sanhueza, 2015).

Las propuestas pedagógicas deben tener como punto de partida el reconocimiento de la diversidad como algo positivo y enriquecedor, abandonando, en consecuencia, la visión de la diversidad cultural como "problemática" y "deficitaria". Este cambio tiene importantes 
implicaciones para el aprendizaje de los escolares, ya que los profesores suelen atribuir el fracaso escolar a las propias capacidades del alumnado y a su situación familiar; es decir, en general manifiestan poca confianza en su propia influencia sobre los logros de sus estudiantes.

La CCI integra una dimensión personal y social (Sanhueza, Cardona y Friz, 2015). Para favorecer esta última dimensión los currículos de formación inicial de profesores debieran incluir temas y/o contenidos donde se les instruya sobre técnicas y estrategias para la coordinación con las familias, el entorno cultural y social que rodea a las escuelas, más aún pensando en que la educación que hoy se requiere traspasa las barreras de la escuela y lo sitúa en una sociedad dinámica y plural.

La literatura (Neuliep, 2012; Huang, 2014; Jiao Xue, 2014) indica que la educación en competencias interculturales integra una importante dimensión afectiva y ética. En este contexto, resulta necesaria la introducción de contenidos sobre valores dentro de las planificaciones. Pueden ser estrategias efectivas; la negociación, técnicas de resolución de conflictos, actividades donde se les invite a ponerse en el lugar del otro, favoreciendo la empatía, confrontar ideas propias con las de sus compañeros, especialmente relativas a las diferencias culturales, entre otras.

Como se ha visto, la CCI es un eje fundamental para la promoción de escuelas inclusivas, de allí la necesidad de que los futuros profesores sean formados en esta materia.

Para futuros trabajos sería recomendable ahondar en aspectos de la comunicación intercultural desde una metodología cualitativa con el propósito de profundizar los resultados obtenidos en la encuesta. Este trabajo posee un carácter exploratorio y, en consecuencia, la generalización de los resultados queda limitada a la muestra presentada y/o a muestras con características similares.

\section{REFERENCIAS BIBLIOGRÁFICAS}

Akkari, A. (2009). Introduction aux approches interculturelles en éducation. Genève : Université de Genève.

(2006). "Les approches multiculturelles dans la formation des enseignants: entre recherche et pédagogie critique". En Revue des HEP de la Suisse romande et du Tessin, vol. 4, Suiza: Conférence des directeurs des Hautes écoles pédagogiques et institutions assimilées de Suisse romande et du Tessin.

Aleksandrovna, L. (2014). Communicative Competency in Teacher Training, World Applied Sciences Journal, 31 (4), 583-586.

Ang, S., Van Dyne, L., Koh, C., Yee, K., Templer, K., Tay, C. \& Chandrasekar, N. (2007). Cultural Intelligence: Its Measurement and Effects on Cultural Judgment and Decision Making, Cultural Adaptation and Task Performance. Management and Organization Review 3(3), 335-371.

Barra, N. (2007). El desafío de la diversidad cultural para Chile. Intercultural Center for Research in Education. Disponible en http://www.incre.org/pdf/Chile.pdf

Cabrera, F., Espín, J. V., Marín, M. y Rodríguez, M. (1999). La formación del profesorado en educación multicultural. En M. A. Essomba (Coord.), Construir la escuela intercultural. Reflexiones y propuestas para trabajar la diversidad étnica y cultural. Barcelona: Graó.

Cardona, M.C. (2002). Introducción a los métodos de investigación en educación. Madrid: EOS.

Cornejo, J., Sanhueza, S. y Rioseco, M. (2012). Orientaciones para la elaboración de tesis, seminarios y paper académicos. Chile: Universidad Católica del Maule. 
Dasen, P. (2002). “Approches interculturelles: acquis et controverses”. En Dasen, Pierre y Perregaux, Christiane (eds.), Pourquoi des approches interculturelles en sciences de l'éducation? Belgique, De Boeck Supérieur.

Dietz, G. y Mateos, L. (2011). Interculturalidad y Educación Intercultural en México: Un análisis de los discursos nacionales e internacionales en su impacto en los modelos educativos mexicanos. Secretaría de Educación Pública. México, D.F.

Dion, S. (2007). "Disrupting molded images: Identities, responsibilities and relationships-teachers and indigenous subject material". En Teaching Education, vol. 18, núm. 4, Australia: University of Queensland.

Gaché, J. (2013). "Éxitos y fracasos de una propuesta educativa basada sobre el” En Método Inductivo Intercultural implementada en el Perú, México y el Brasil. Inclusión Social y Equidad en la Educación Superior, núm. 13, Chile: Fundación Equitas.

Gay, G. y Tyrone H. (2000). "Multicultural teacher education for the 21st century". En The Teacher Educator, 36 (1), 1-16.

Huang, Y. (2014). Constructing Intercultural Communicative Competence Framework for English Learners. Cross-Cultural Communication, 10 (1), 97-101.

Jiao, X. (2014). Cultivating Intercultural Communication Competence through Culture Teaching. Theory and Practice in Language Studies, 4 (7), 1492-1498.

Jordán, J. (1996). Propuestas de educación intercultural para profesores. Barcelona, CEAC.

Jordán, J., Castellá, E. y Pinto, C. (2001). La educación intercultural, una respuesta a tiempo. Barcelona: UOC.

Lacourse F. y Maubant P., (2009). L'intervention éducative: un cadre conceptuel pertinent pour une lecture compréhensive de la pratique enseignante. Introduction Nouveaux cahiers de la recherche en éducation, 12 (1), 3-8.

Lázár I., Huber-Kriegler, M., Lussier, D., Matei, G. y Peck, Ch. (2007). Développer et évaluer la compétence en communication interculturelle. Un guide à l'usage des enseignants de langues et des formateurs d'enseignants. Centre européen pour les langues vivantes / Conseil de l'Europe.

Leiva, J. (2012). La formación en educación intercultural del profesorado y la comunidad. Revista Electrónica de Investigación y Docencia, número monográfico, 8-31.

Lipiansky, E. (1992). Identité et communication. París: Presses Universitaires de France.

López, M. (2006). Cultura de la diversidad, cultura de la inclusión: educar para construir una escuela sin exclusiones. In VV. AA.., L'Ecola que inclou (pp. 11-52), (Collecction Hort de Trenor 18). Torrent: Ajuntament de Torrent.

Losada, J. y López, R. (2003). Métodos de investigación en ciencias humanas y sociales. España: Ediciones Paraninfo.

Mampaey, J. y Zanoni, P. (2015). "Reproducing monocultural education: ethnic majority staff's discursive constructions of monocultural school practices". En British Journal of Sociology of Education, (ahead-of-print), Inglaterra: London School of Economics.

Muller, M., Calcagni, E., Grau, V., Preiss, D. y Volante, P. (2013). Desarrollo de habilidades de observación en estudiantes de pedagogía: Resultados de una intervención piloto basada en el uso de la Videoteca de Buenas Prácticas Docentes. Estudios pedagógicos 39, nº especial, 85-101.

Neuliep, J. (2012). The Relationship among Intercultural Communication Apprehension, Ethnocentrism, Uncertainty Reduction, and Communication Satisfaction during Initial Intercultural Interaction: An Extension of Anxiety and Uncertainty Management (AUM) Theory. Journal of Intercultural Communication Research, 41 (1), 1-16.

Ouellet, F. (1991). L'éducation interculturelle. Essai sur le contenu de la formation des maîtres. Paris: Editions L'Harmattan.

Ouellet, F. (2002). L' education interculturelle et L'education a la citoyenneté. Quelques pistes pour s'orienter dans la diversité des conceptions. VEI Enjeux, 129, 146-167.

Quilaqueo, D. y Quintriqueo, S. (2010). Saberes educativos mapuches: Un análisis desde la 
perspectiva de los Kimches. Revista de la Universidad Bolivariana, 9 (26), 337-360.

Quilaqueo, D., Quintriqueo, S., Torres, H. y Muñoz, G. (2014), "Saberes educativos mapuches: aportes epistémicos para un enfoque de educación intercultural". Revista Chungará, 46 (2), 271-283.

Quilaqueo, D., Quintriqueo, S., Torres, H. (2015). Características epistémicas de los métodos educativos mapuches. Revista Electrónica de Investigación Educativa (aceptado).

Quintriqueo, S., Quilaqueo, D., Peña-Cortés, F. y Muñoz, G. (2015). Conocimientos culturales como contenidos de la educación familiar mapuche. Revista ALPHA, $\mathrm{N}^{\circ} 40,131-146$.

Quintriqueo, S., Quilaqueo, D., Gutiérrez, M., Morales, S. y Susan S. (2015). Valores educativos mapuches: base para la educación y comunicación intercultural en la formación docente. En Valdebenito V. y Mellado, M. (2015). Gestión escolar: liderazgo y acción pedagógica. Temuco: Editorial Universidad Católica de Temuco.

Ragoonaden, K. (2011). La compétence interculturelle et la formation initiale: le point sur le CECR et l'IDI. Revue canadienne de linguistique appliquée, numéro hors-série, 14 (2), 86-105.

Sanhueza, S. Cardona, M.C. y Friz, M. (2015). Understanding elementary and secondary students' representation of cultural differences as reflected in the process of intercultural communication in school contexts, Nordic Psychology, 67 (1), 65-86.

Sanhueza, S.; Friz, M. y Quintriqueo, S. (2014). Estudio exploratorio sobre las actitudes y comportamiento del profesorado de Chile en contextos de escolarización de alumnado inmigrante. Revista Electrónica de Investigación Educativa, 16(3), 148-162.

Schütz, A. (1979). Estudios sobre teoría social. Buenos Aires: Amorrortu.

Steinbach, M. (2012). Élargir les perspectives interculturelles des futurs enseignants. Revue des sciences de l'éducation de McGill, 47 (2), 153-170.

Valiente, T. (2001), "Reflexiones en torno a interculturalidad y proceso educativo en sociedades multiculturales: encanto y desafío de la diversidad". Lexis, 25 (1-2), 337-365. 